\title{
Electrochemical Characterization of Carbon Nanopipette Electrodes for Rapid Dopamine Detection
}

\author{
Hillary Renee Rees \\ Mount Crawford, Virginia
}

B.S., Bridgewater College, 2012

\begin{abstract}
A Thesis presented to the Graduate Faculty
of the University of Virginia in Candidacy for the Degree of Master of Science
\end{abstract}

Department of Chemistry

University of Virginia

May, 2014 


\section{Electrochemical Characterization of Carbon Nanopipette Electrodes for Rapid Dopamine Detection}

\section{Abstract}

Dopamine is a neurotransmitter involved in motor, reward, and cognitive functions. Model systems are useful for studies of dopamine-related diseases, such as Parkinson's and schizophrenia. Drosophila melanogaster is a convenient model organism because it has homologous neurotransmitters with mammals and is easy and fast for genetic manipulation. However, the central nervous system is extremely small, making real-time measurements of dopamine difficult. In the past, carbon fiber microelectrodes and fast-scan cyclic voltammetry (FSCV) have been used to detect dopamine release in the Drosophila ventral nerve cord. In order to measure dopamine in specific brain regions of the fly, a smaller, robust electrode is needed.

Carbon nanopipette electrodes (CNPEs) have been made with hollow tips for concurrent electrophysiological measurement and injection. The CNPEs used in this work are specifically designed to have solid tips 50-400 $\mathrm{nm}$ in diameter. This thesis introduces the use of CNPEs for detecting changes in concentrations of dopamine using FSCV. Chapter 1 describes CNPEs, gives a brief overview of neurotransmission and electrochemistry, and explains FSCV. Chapter 2 explores the characterization of the electrochemical properties of CNPEs using FSCV. We verified these electrodes were suitable for in vivo studies by measuring exogenous and endogenous dopamine in Drosophila larvae. Chapter 3 describes future studies that can be done with CNPEs. Ultimately, we envision using CNPEs in the adult Drosophila to study specific regions of the fully developed central nervous system. 


\section{Table of Contents}

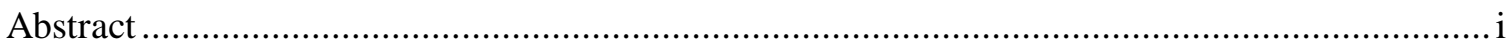

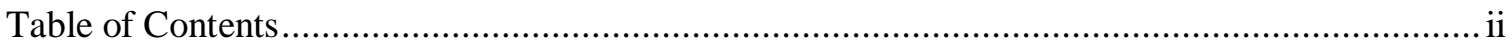

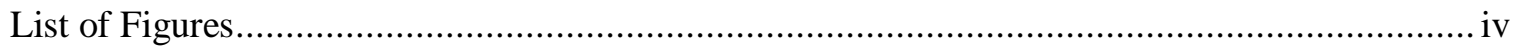

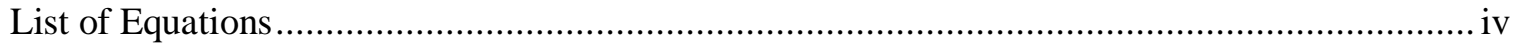

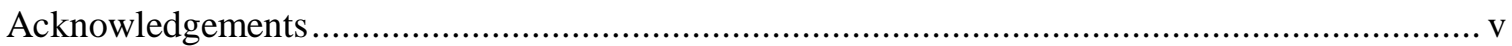

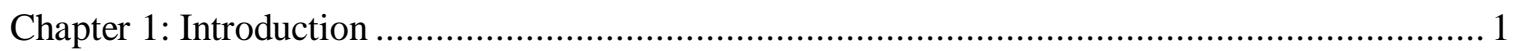

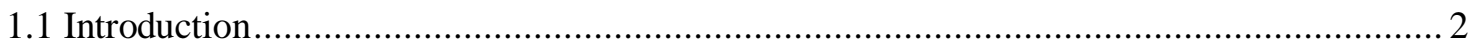

1.1.1 Nanoelectrodes for Electrochemical Applications ................................................... 3

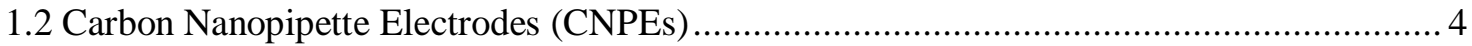

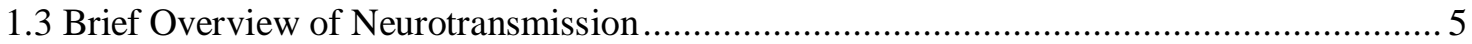

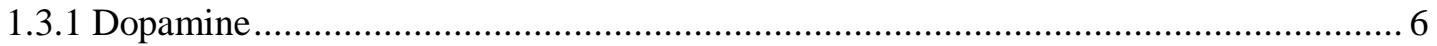

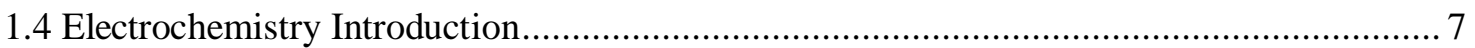

1.5 Fast-Scan Cyclic Voltammetry (FSCV) .............................................................. 9

1.5.1 Using FSCV to characterize CNPEs and detect dopamine in Drosophila .................. 11

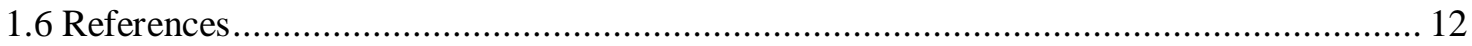

Chapter 2: Electrochemical Characterization of Carbon Nanopipette Electrodes for Rapid

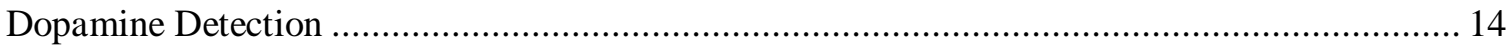

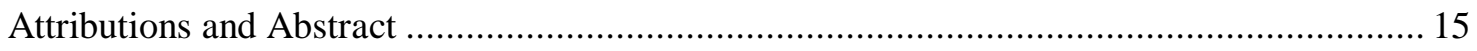

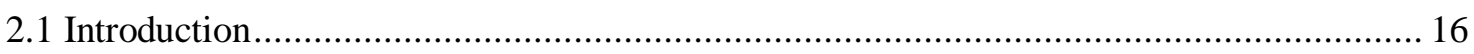

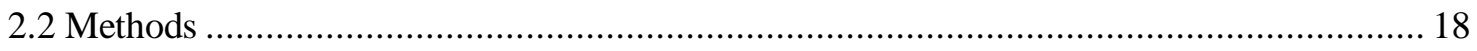

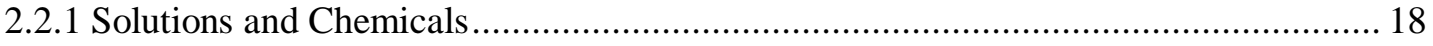

2.2.2 Carbon Nanopipette Electrode Fabrication.......................................................... 18

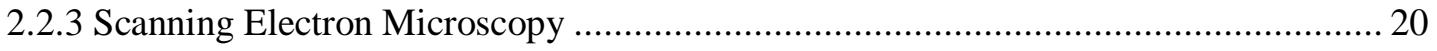

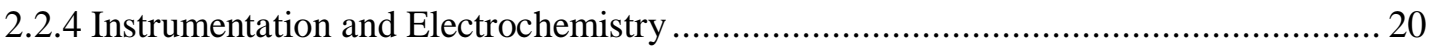

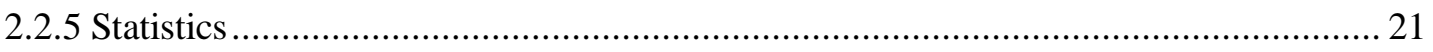

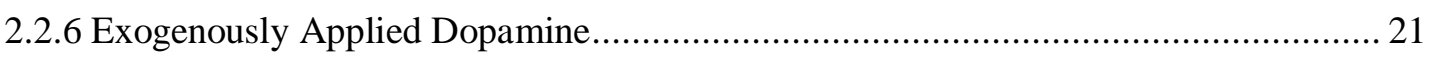

2.2.7 Endogenous Dopamine Evoked by Chrimson Channelrhodopsin Stimulation............ 21

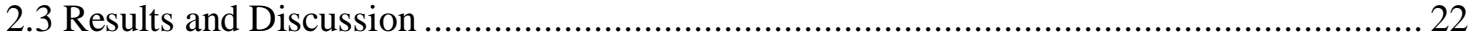

2.3.1 Fabrication of Carbon Nanopipette Electrodes …...................................................... 22 


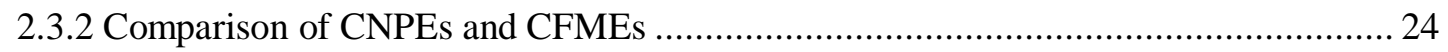

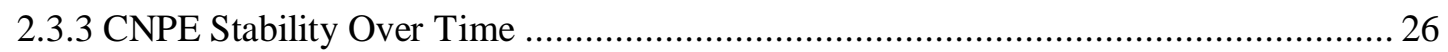

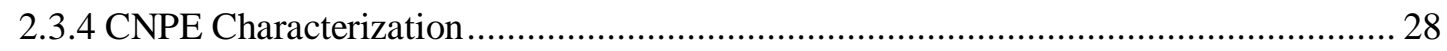

2.3.5 Measurements of Exogenous Dopamine in Drosophila ...........................................2 29

2.3.6 Measurements of Endogenous Dopamine in Drosophila Evoked by Chrimson

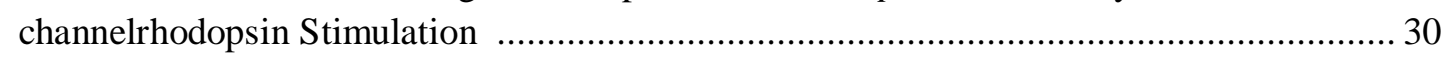

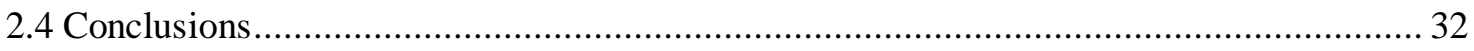

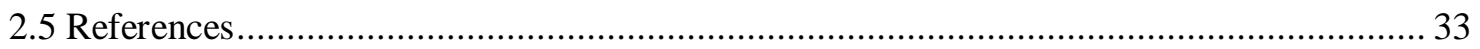

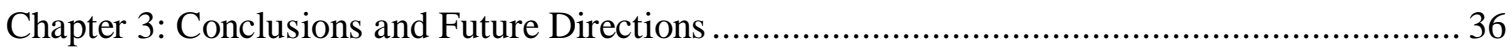

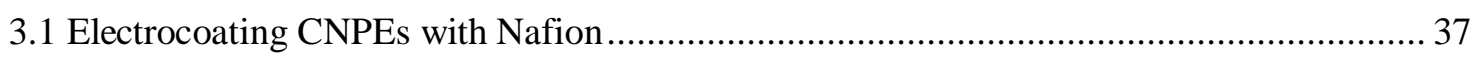

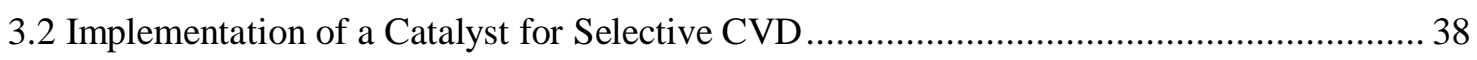

3.3 Direct Measurements from Drosophila Mushroom Bodies ............................................ 38

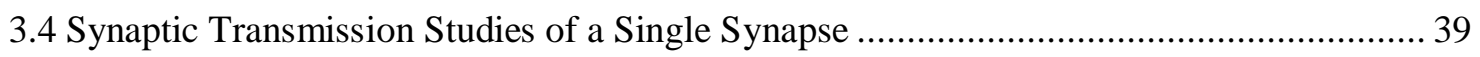

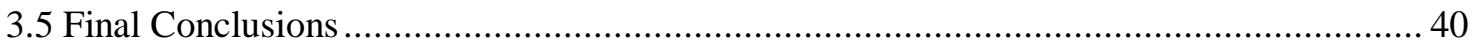

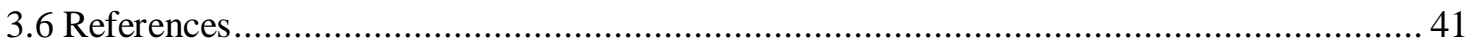




\section{List of Figures}

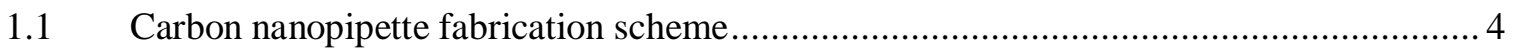

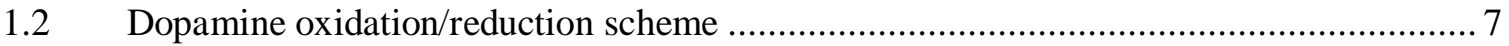

1.3 Typical FSCV waveform used with CNPs for dopamine detection ............................... 9

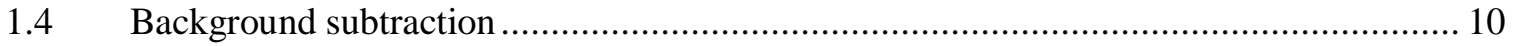

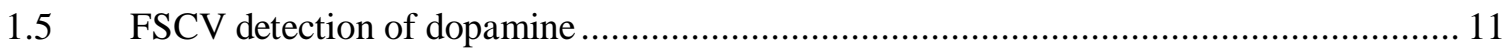

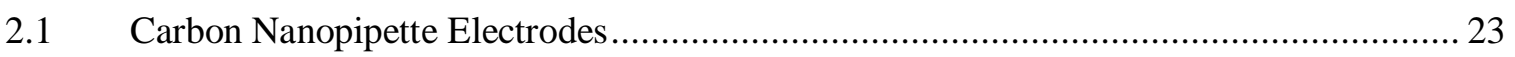

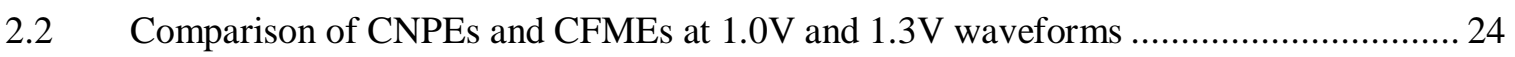

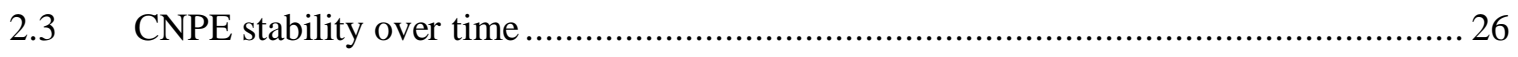

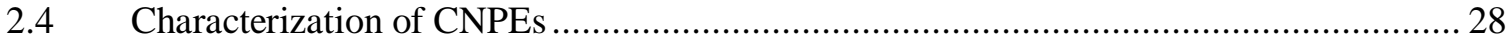

2.5 Measurements of exogenous dopamine in a Drosophila CNS ................................... 29

2.6 Measurements of endogenous dopamine evoked by Chrimson ................................... 31

\section{List of Equations}

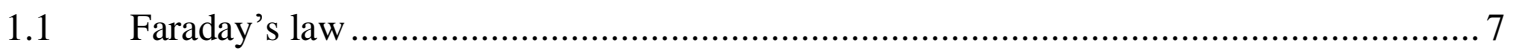

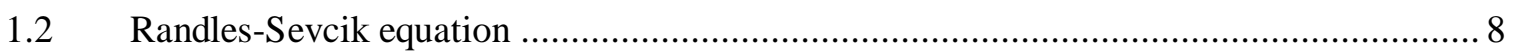

1.3 Voltammetry for a nernstian adsorbate layer ........................................................... 9 


\section{Acknowledgements}

First and foremost I would like to thank my parents. Their unconditional love and support is how I was able to keep my head up and push forward to write this thesis. I couldn't have done it without you. I would also like to thank Dr. Venton for all her time spent editing this work. Hopefully I have become a better writer. Thank you to Dr. Sara Fitzgerald who was always willing to meet up with me just to listen and gave me great words of encouragement. This journey was one I needed to experience. 


\section{Chapter 1}

\section{Introduction}




\subsection{Introduction}

The overall goal of this work was to use fast-scan cyclic voltammetry (FSCV) for electrochemical characterization of carbon nanopipette electrodes (CNPEs). FSCV allows for sub-second time resolution needed to monitor fast concentration changes in neurotransmitters. CNPEs provide spatial resolution because of the nano-scale tip diameter. With FSCV and CNPEs, we have a new probe small and fast enough to study neurotransmission in Drosophila melanogaster. This introduction will briefly discuss a few analytical techniques used to study neurotransmission, discuss nanoelectrodes for electrochemical applications, introduce carbon nanopipette electrodes, give an overview of neurotransmission and electrochemistry, and describe FSCV.

There are several methods to study neurotransmission such as microdialysis, amperometry, and FSCV. In microdialysis, a sample of extracellular fluid of the brain is taken and small molecules are recovered. ${ }^{1}$ Samples are then analyzed by other analytical techniques such as chromatography or capillary electrophoresis. This coupling allows for high selectivity and sensitivity for almost any neurotransmitter. However, microdialysis is not useful for real-time measurements of neurotransmitters because of its low temporal resolution $(\sim 600 \mathrm{~s}){ }^{1}$

Constant-potential amperometry measures current continuously allowing for high temporal resolution (as fast as $\sim 100 \mu \mathrm{s}$ ). ${ }^{2}$ A constant voltage is applied to oxidize the molecule of interest. Amperometry is usually used for single cell studies where the electroactive molecule is known because there is no chemical resolution. Any molecule that undergoes a redox reaction at the applied potential is detected, which is not desirable for studies of complex environments in vivo. 
Fast-scan cyclic voltammetry provides a sensitive and selective way of taking measurements in vivo. FSCV does not have the temporal resolution of amperometry, but does allow for chemical identification. Most commonly used with FSCV are carbon fiber microelectrodes (CFMEs) which are approximately 5-10 $\mu \mathrm{m}$ in diameter, more than 20 times smaller than typical microdialysis probes. CFMEs can be placed within micrometers of neuronal terminals and cause very little damage when inserted in tissue. ${ }^{3}$ In the past, CFMEs have been used to measure dopamine release in Drosophila larvae using FSCV. ${ }^{4}$ However, even with this micron-scale electrode, there is a push to produce even smaller electrodes for better spatial resolution to study specific regions of the brain. Such electrodes will be useful for studying the fruit fly in particular because its CNS is only $100 \mu \mathrm{m}$ wide. ${ }^{4}$

\subsubsection{Nanoelectrodes for Electrochemical Applications}

Over the past few decades, nanoelectrodes have been developed for electrochemical applications. Carbon electrodes in particular provide many advantages such as low cost, wide potential window, and good adsorption properties. ${ }^{5}$ Some carbon nanoelectrodes are made by electrochemical or flame etching carbon fibers to sub-micron tips. $^{6-9}$ Other methods deposit electrophoretic paint to completely insulate an etched carbon fiber except for the very tip leaving an effective diameter of a few nanometers. ${ }^{10,11}$ Carbon nanomaterials have also been incorporated into nano electrochemical sensors. Individual single-walled nanotube sensors have been fabricated but on silicon wafers, ${ }^{12}$ not easily implantable for in vivo studies. Multiwalled carbon nanotubes have also been used as an electrochemical probe, but again require extensive cutting and insulation. ${ }^{13}$ 
For in vivo measurements in Drosophila, we desire a sharp, carbon nanoelectrode with high sensitivity to detect nanomolar concentrations and a facile fabrication.

\subsection{Carbon Nanopipette Electrodes (CNPEs)}

Hiam Bau's group at the University of Pennsylvania first fabricated carbon nanopipettes in 2008 with open tips primarily for facilitating electrical measurements while delivering fluids into cells. ${ }^{14,15}$ Figure 1.1 shows the schematic of CNPE fabrication. Quartz capillaries are pulled into two fine-tipped micropipettes (Figure 1.1 (i)). Carbon is then deposited on the inner surface by chemical vapor deposition (CVD) with methane and argon (Figure 1.1 (ii)) forming a hollow, conductive channel. At a high temperature, usually $900^{\circ} \mathrm{C}$, the methane gas decomposes to hydrocarbons which deposit selectively on the inner surface. The thickness of this

(i)

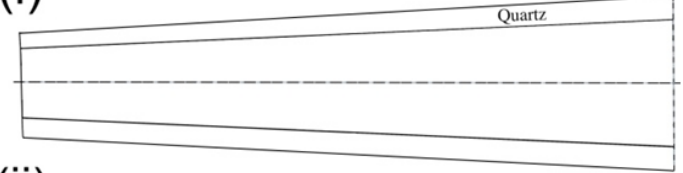

(ii)
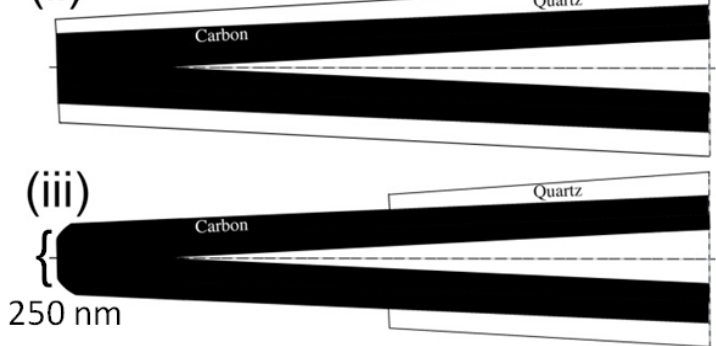

Figure 1.1. Carbon nanopipette fabrication scheme. (i) Pulled micropipette. (ii) Deposition of carbon by CVD. (iii) Exposing carbon tip by wet-etching.

film is controlled by the CVD time. The

tip of the micropipette is then wet-etched with HF to remove the quartz and leave an exposed tip of carbon (Figure 1.1 (iii)). The length of the exposed carbon is controlled by the etching time and temperature. This process allows for batch fabrication of hundreds of probes with nanoscale dimensions. For our work, the CNPEs were made specifically with a solid tip. To get a solid, closed tip, the CVD time is greater than traditional CNPEs. 
The primary advantage of carbon nanopipette electrodes is their small size. The average CNPE, used in our work, is $200-400 \mathrm{~nm}$ in diameter at the tip and has an exposed carbon length of $\sim 150 \mu \mathrm{m}$. Because of this small size, we hypothesize CNPEs will cause less tissue damage than traditional CFMEs. The dagger-like shape allows for a robust electrode that can penetrate Drosophila larvae easily. CNPEs have shown to have electrochemical properties similar to CFMEs. This will be described further in Chapter 2 . In solution and at higher potentials, the carbon-carbon bonds of the electrode break, oxidizing the surface with functional groups such as phenols, carboxyl groups and ketones. ${ }^{16}$ Oxide groups are advantageous because these negatively charged groups are more selective for positively charged molecules such as dopamine. CNPEs are small and fast enough for studying mechanisms of neurotransmitter release.

\subsection{Brief Overview of Neurotransmission}

Cells communicate by passing signals through the body, a process called neurotransmission. Synthesis of the neurotransmitter takes place in the cell body or terminals. The neurotransmitters are then packaged and stored in vesicles. ${ }^{17}$ Sodium ions move through the cell membrane ion channels causing depolarization, resulting in an action potential. During an action potential the neurotransmitters are released into the synapse, the space between the axon terminal of the presynaptic neuron and the dendrite of the postsynaptic neuron. From here, a few different things can happen. The neurotransmitters can diffuse across the synapse and bind to postsynaptic receptors on the dendrite. Neurotransmitters can also bind to presynaptic transporters clearing them from the extracellular space and regulating further release. Some neurotransmitters may also 
diffuse out of the synapse and remain in the extracellular space or be degraded by enzymes. $^{18}$

The synapse is only $20-40 \mathrm{~nm},{ }^{19}$ so currently a CNPE is not able to measure only in one synapse. All measurements come from the extracellular space and still provide useful information. Volume transmission occurs in the extracellular space and consists of diffusion of signals for distances longer than the synaptic cleft. ${ }^{20}$ Measuring in the extracellular space is advantageous when studying certain diseases and neurotransmitters. For example, in a model of Parkinson's disease, volume transmission still occurs in dopamine nerve terminals. ${ }^{21}$ Serotonin and dopamine are two chemical mediators involved in volume transmission. ${ }^{22}$

\subsubsection{Dopamine}

The first neurotransmitter of study with FSCV is usually dopamine (DA). Dopamine is involved in motor, reward, and cognitive functions. Parkinson's disease is linked to reduced levels of dopamine; whereas, an excess of dopamine may cause psychiatric problems. ${ }^{23}$ At sufficient potentials, dopamine undergoes a two electron oxidation to dopamine-ortho-quinone (DOQ) (Figure 1.2). Dopamine diffuses towards the electrode, where it adsorbs to the surface. When a positive voltage is applied to the electrode, dopamine loses two electrons oxidizing to DOQ. This DOQ can desorb into solution or be reduced back to dopamine when the potential applied to the electrode is switched back to a negative potential. 


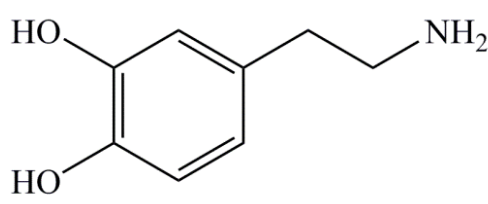

Dopamine (DA)
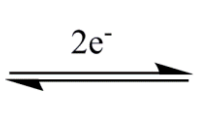

Dopamine-ortho-quinone (DOQ)

Figure 1.2. Dopamine undergoes a two electron oxidation at the cis-diol to dopamine-orthoquinone. DOQ can reduce back to DA.

\subsection{Electrochemistry Introduction}

Electrochemistry is a valuable technique for measuring fast reactions. Given an applied electrical potential, an analyte will oxidize (lose electrons) or reduce (gain electrons). Electrochemical processes involve electron transfer at an electrode surface during the redox reaction. The movement of electrons, or current, is measured at the working electrode. Electrochemical techniques are used for studying a variety of analytes. Electrochemical sensors can detect antibiotic contaminants in water at a sensitivity at 0.001 parts per trillion. ${ }^{24}$ At home glucometers utilize disposable electrochemical cells and measure blood glucose levels through the oxidation of glucose. ${ }^{25}$ In the Venton lab, we utilize electrochemistry to study neurotransmitters.

In a FSCV system, the potential between the working and reference electrodes is controlled and a potentiostat measures and amplifies the resulting current at the working electrode. The current, or charge flow over time, from the redox reactions is called Faradaic current and is proportional to the number of moles reacted (Equation1.1)

$Q=n F \Delta N$

$Q=$ charge passed across the electrode

$n=$ number of electrons transferred

$F=$ Faraday's constant

$\Delta N=$ number of moles reacted 
There is another type of current that occurs called nonfaradaic current. This is a result of the polarization of the surrounding solution. When a positive potential is applied to the electrode, positively charged ions are repelled and negative ions move toward the electrode setting up what is called a double layer. There is a negatively charged layer specifically adsorbed to the electrode, called the inner Helmholtz layer. This layer attracts cations which form the outer Helmholtz layer. The separation and flow of the ions cause nonfaradaic, or charging, current. All measurements have a charging current and therefore a background signal.

Diffusion to the electrode is generally assumed to be the rate-limiting step for electrochemical detection. The kinetics are described by Equation 1.2. From this equation, there is a linear relationship between peak current and the square root of scan rate.

$i_{p}=\left(2.69 * 10^{5}\right) n^{3 / 2} A D^{1 / 2} C v^{1 / 2}$ Equation 1.2

$i_{p}=$ peak current $(\mathrm{A})$

$n=$ number of moles

$A=\operatorname{area}\left(\mathrm{cm}^{2}\right)$

$D=$ diffusion coefficient $\left(\mathrm{cm}^{2} / \mathrm{s}\right)$

$C=$ concentration $\left(\mathrm{mol} / \mathrm{cm}^{2}\right)$

$v=$ scan rate $(\mathrm{V} / \mathrm{s})$

Sometimes the adsorption of the electroactive species to the electrode is rate limiting because of the interaction with the electrode surface functional groups. This limits the rate of the reaction and is described by Equation 1.3. There is a linear relationship between peak current and scan rate. 
$i_{p}=\frac{n^{2} F^{2}}{4 R T} v A \Gamma_{o}$

Equation 1.3

$F=$ Faraday's constant $\left(9.64853 * 10^{4} \mathrm{C}\right)$

$R=$ Gas constant $(8.31447 \mathrm{~J} / \mathrm{molK})$

$T=$ temperature $(\mathrm{K})$

$\Gamma_{o}=$ surface coverage $\left(\mathrm{mol} / \mathrm{cm}^{2}\right)$

\subsection{Fast-Scan Cyclic Voltammetry (FSCV)}

In FSCV, voltage is applied to a working electrode through a range of potentials in a triangular waveform, as shown in Figure 1.3.

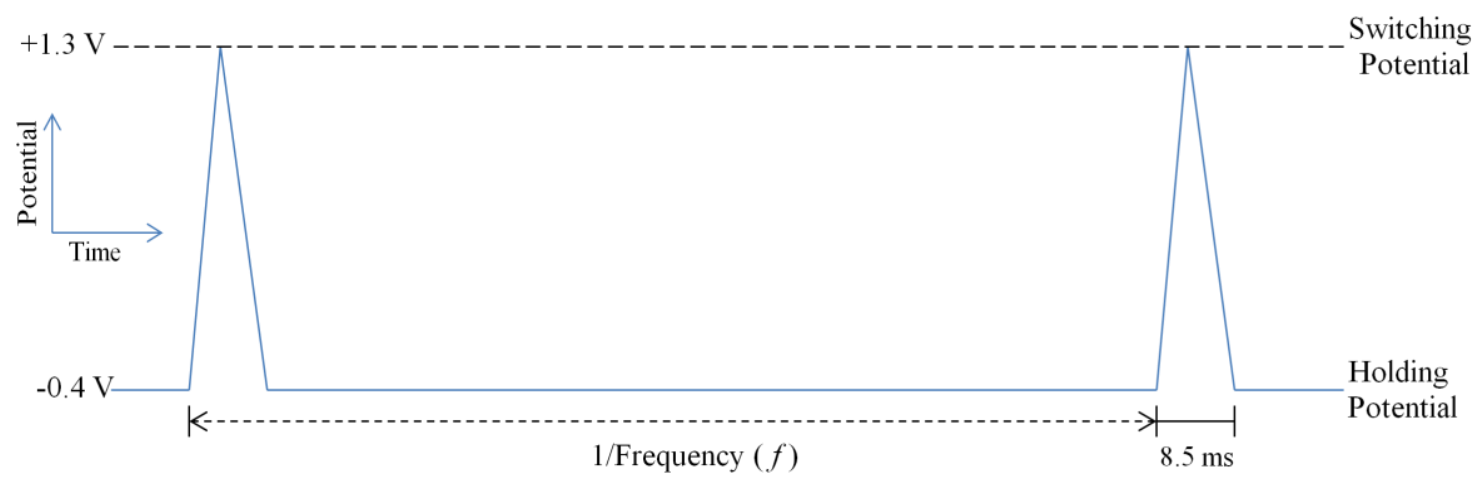

Figure 1.3. A typical FSCV waveform used with CNPEs for dopamine detection.

A positive potential is applied to the electrode during the upward scan. During oxidation, a molecule loses one or more electrons to the positive electrode. During the downward scan, the electrode returns to its negative holding potential and molecules are reduced. It is this movement of electrons during oxidation and reduction that is the measured Faradaic current. The ramp is linear from the holding potential $(-0.4 \mathrm{~V})$ up to the switching potential $(1.3 \mathrm{~V})$ usually at a rate of $400 \mathrm{~V} / \mathrm{s}$; therefore, one scan takes only 8.5 ms. The charging current is much larger than faradaic current and overshadows the oxidation and reduction peaks of raw cyclic voltammograms (CVs). To only obtain measurements from the desired Faradaic current, the charging current is background subtracted resulting in a clear CV of dopamine (Figure 1.4). Due to the background 
current being so large and background subtraction, changes in concentrations are measured rather than just basal concentrations. ${ }^{26}$
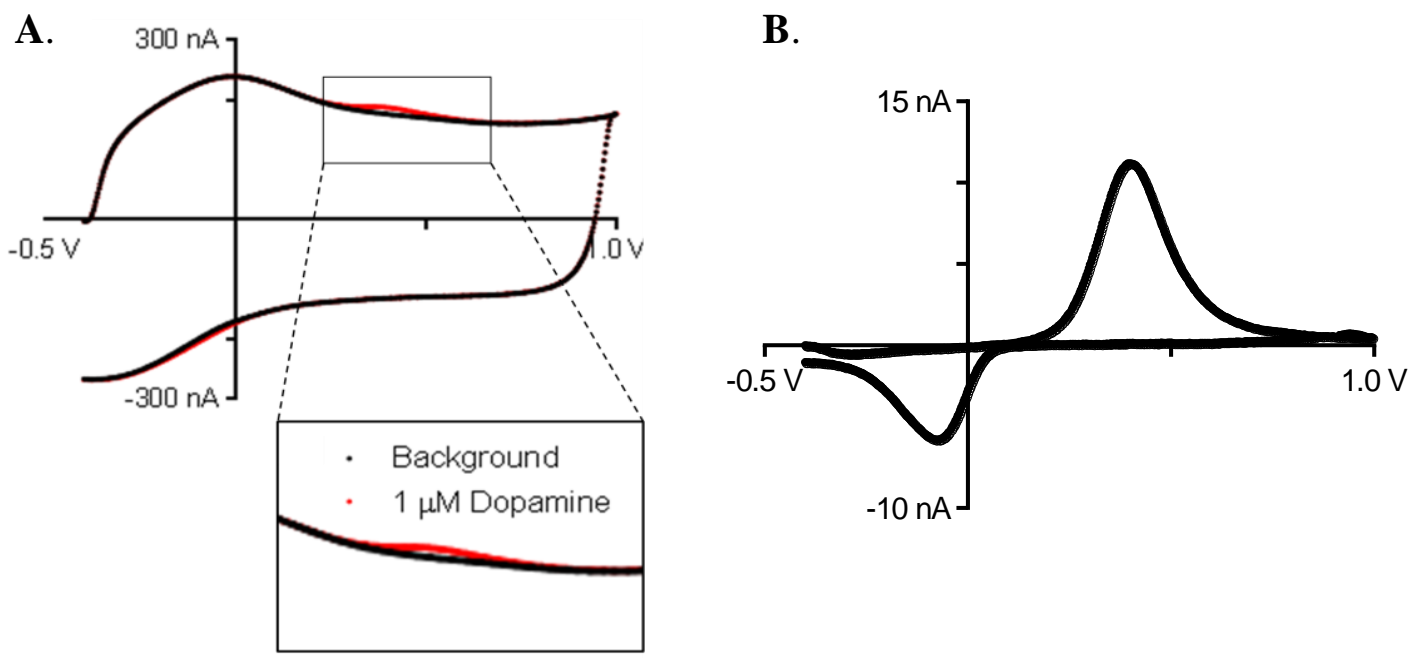

Figure 1.4. (A) Overlaid currents with buffer only (black) and injected $1 \mu \mathrm{M}$ dopamine (red). The background current is subtracted from the sample current resulting in a cyclic voltammogram (B). Data collected with a CNPE.

All data collected during a trial is represented in a color plot as shown in Figure 1.5. During the first five seconds, only buffer is injected. From 5-10 seconds a known concentration of dopamine in buffer is injected. For the last five seconds the flow is switched back to just buffer. The current versus time plot is a horizontal slice at a given voltage. A CV is a vertical slice of the color plot at a given time showing the relationship between voltage and current. This ability to obtain a characteristic CV gives FSCV a major advantage over other electrochemical detection methods. 
A.

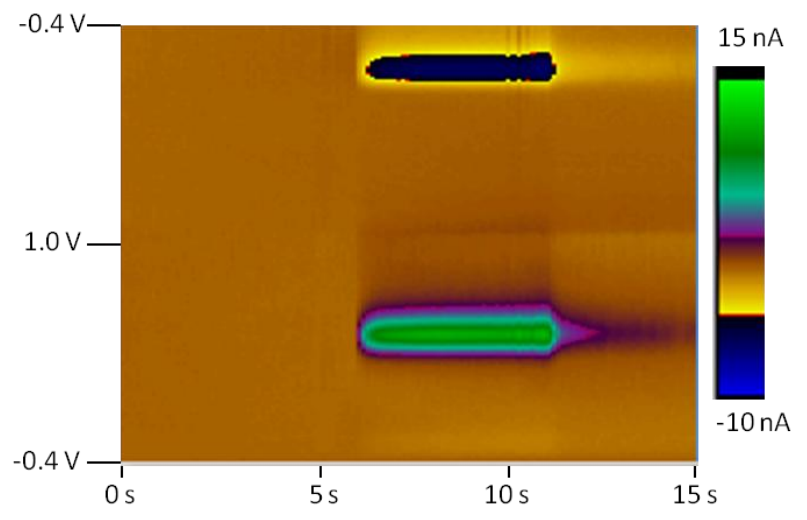

B.
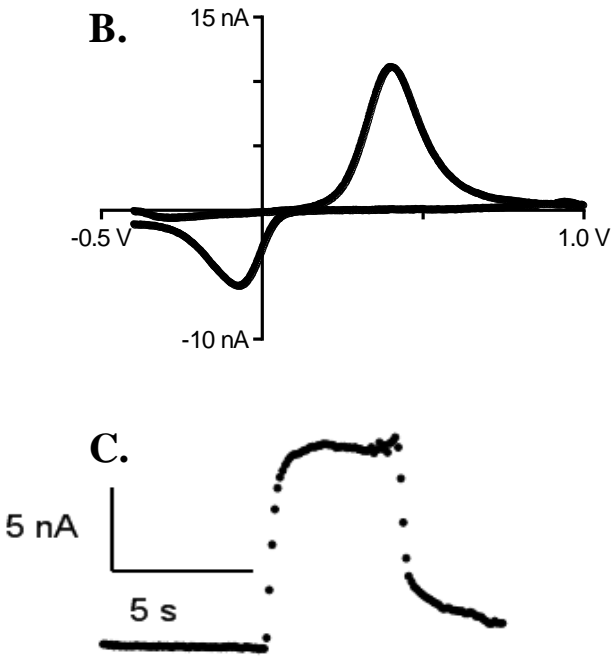

Figure 1.5. FSCV detection of dopamine. (A) An example color plot resulting from a $5 \mathrm{~s} 1 \mu \mathrm{M}$ dopamine injection. Current is shown in false color. The cyclic voltammogram (B) is taken at approximately $8 \mathrm{~s}$ and a current versus time plot $(\mathrm{C})$ for the oxidation.

\subsubsection{Using FSCV to characterize CNPEs and detect dopamine in Drosophila}

Drosophila is a model organism for studying dopamine signaling, but the small size presents challenges for neurotransmission studies. The size of CNPEs allows for good spatial resolution in vivo, and FSCV is adventageous for sensitive, selective, realtime measurements. In this thesis, CNPEs are used with FSCV for the first time to detect changes in dopamine levels. The goal is to characterize these CNPEs with FSCV and optimize parameters so measurements can be made in vivo to study dopamine effects due to diseases, behavior, or genetics. Chapter 2 describes the research conducted and the results obtained, to determine the electrochemical properties of CNPEs. Also, the data verify CNPEs can detect dopamine in Drosophila larvae. Chapter 3 suggests future directions that can be taken with CNPEs and FSCV. Ultimately, we envision using CNPEs in the adult Drosophila to study specific regions of the fully developed central nervous system and provide growth in the field so that new functions of the brain can be studied. 


\subsection{References}

1. Watson, C. J.; Venton, B. J.; Kennedy, R. T. In Vivo Measurements of Neurotransmitters by Microdialysis Sampling. Analytical Chemistry 2006, 1391-1399.

2. Zhang, B.; Heien, M. L.; Santillo, M. F.; Mellander, L.; Ewing, A. Temporal Resolution in Electrochemical Imaging on Single PC12 Cells Using Amperometry and Voltammetry at Microelectrode Arrays. Analytical Chemistry 2011, 83, 571-577.

3. Peters, J.; Miner, L.; Michael, A. C.; Sesack, S. Ultrastructure of carbon fiber microelectrode implantation sites after acute voltammetric measurements in the striatum of anesthetized rats. Journal of Neuroscience Methods 2004, 137 (1), 9-23.

4. Vickrey, T. L.; Condron, B.; Venton, B. J. Detection of Endogenous Dopamine Changes in Drosophila melanogaster Using Fast-Scan Cyclic Voltammetry. Analytical Chemistry 2009, 81, 9306-9313.

5. McCreery, R. L. Advanced Carbon Electrode Materials for Molecular Electrochemistry. Chem. Rev. 2008, 108, 2646-2687.

6. Huang, W.; Pang, D.; Tong, H.; Wang, Z.; Cheng, J. A Method for the Fabrication of Low-Noise Carbon Fiber Nanoelectrodes. Analytical Chemistry 2001, 73, 1048-1052.

7. Strand, A. M.; Venton, B. J. Flame Etching Enhances the Sensitivity of Carbon-Fiber Microelectrodes. Analytical Chemistry 2008, 80, 3708-3715.

8. Kawagoe, K. T.; Jankowski, J. A.; Wightman, R. M. Etched Carbon-Fiber Electrodes as Amperometric Detectors of Catecholamine Secretion from Isolated Biological Cells. Analytical Chemistry 1991, 63, 1589-1594.

9. Strein, T. G.; Ewing, A. Characterization of Submicron-Sized Carbon Electrodes Insulated with a Phenol-Allylphenol Copolymer. Analytical Chemistry 1992, 64, 1368-1373.

10. Chen, S.; Kucernak, A. Fabrication of carbon microelectrodes with an effective radius of $1 \mathrm{~nm}$. Electrochemistry Communications 2002, 4, 80-85.

11. Wang, C.; Chen, Y.; Wang, F.; Hu, X. Fabrication of nanometer-sized carbon electrodes by the controllable electrochemical deposition. Electrochimica Acta 2005, 50, 5588-5593.

12. Heller, I.; Kong, J.; Heering, H. A.; Williams, K. A.; Lemay, S. G.; Dekker, C. Individual Single-Walled Carbon Nanotubes as Nanoelectrodes for Electrochemistry. Nano Letters 2005, 5 (1), 137-142. 
13. Campbell, J. K.; Sun, L.; Crooks, R. M. Electrochemistry Using Single Carbon Nanotubes. J. Am. Chem. Soc. 1999, 121, 3779-3780.

14. Schrlau, M. G.; Dun, N. J.; Bau, H. H. Cell Electrophysiology with Carbon Nanopipettes. ACS NANO 2009, 3 (3), 563-568.

15. Schrlau, M. G.; Falls, E. M.; Ziober, B. L.; Bau, H. H. Carbon nanopipettes for cell probes and intracellular injection. Nanotechnology 2008, 19 (1).

16. McCreery, R. L. Carbon Electrodes: Structural Effects on Electron Transfer Kinetics. In Electroanalytical Chemistry, Bard, A. J., Ed.; Marcel Dekker Inc.: New York, 1991; p 259.

17. Purves, D.; Augustine, G.; Fitzpatrick D; et al. Neuroscience; 2nd ed.; Sinauer Associates: Sunderland (MA), 2001.

18. Kolb, B.; Whishaw, I. In Fundamentals of Human Neuropsychology, 5th ed.; 2003; pp 102-104.

19. Kandel, E. R.; Schwartz, J. H.; Wickens, J. Principles of Neural Science; 4th ed.; McGraw-Hill: New York, 2000.

20. Agnati, L.; Zoli, M.; Stromberg, I.; Fuxe, K. Intercellular communication in the brain: wiring versus volume transmission. Neuroscience 1995, 69 (3), 711726.

21. Zoli, M.; Torri, C.; Ferrari, R.; Jansson, A.; Isabella, Z.; Fuxe, K.; Agnati, L. The emergence of the volume transmission concept. Brain Research Reviews 1998, 26 (2-3), 136-147.

22. Agnati, L.; Guidolin, D.; Guescini, M.; Genedani, S.; Fuxe, K. Understanding wiring and volume transmission. Brain Research Reviews 2010, 64 (1), 137-159.

23. Arbuthnott, G. W.; Wickens, J. Space, time and dopamine. Trends in Neurosciences 2007, 30, 62 .

24. Jacobs, M.; Nagaraj, V. J.; Mertz, T.; Selvam, A. P.; Ngo, T.; Prasad, S. An electrochemical sensor for the detection of antibiotic contaminants in water. Analytical Methods 2013, 5 (17), 4325-4329.

25. Newman, J. D.; Turner, A. P. F. Home blood glucose biosensors: a commercial perspective. Biosensors and Bioelectronics 2005, 20 (12), 2435-2453.

26. Borland, L. M.; Michael, A. C. An Introduction to Electrochemical Methods in Neuroscience. In Electrochemical Methods for Neuroscience, Borland, L. M., Michael, A. C., Eds.; CRC Press: Boca Raton, 2007. 
Chapter 2

\section{Electrochemical Characterization of Carbon Nanopipette Electrodes for Rapid Dopamine Detection}




\title{
Attribution
}

Sean Anderson (University of Pennsylvania) fabricated all carbon nanopipette electrodes and took the SEM images. Eve Privman (University of Virginia) collected all Drosophila data.

\begin{abstract}
Small, robust, sensitive electrodes are desired for in vivo measurements. Carbon nanopipettes have been manufactured and used for drug delivery and electrophysiological measurements. Here, a modified fabrication procedure is used to produce batches of carbon nanopipette electrodes (CNPEs) that have solid tips $\sim 250 \mathrm{~nm}$ in diameter. A pulled quartz capillary was coated with a layer of carbon along the entire inner surface by CVD. The thickness of the layer was controlled to seal the pipette's narrow opening. The quartz tip is then etched with buffered hydrofluoric acid to expose a tapered carbon cylinder 100-150 $\mu \mathrm{m}$ in length. The electrochemical properties of CNPEs are characterized with fast-scan cyclic voltammetry (FSCV) for the first time and the suitability of CNPEs tested for real-time measurements of dopamine in Drosophila. Faster electron transfer kinetics but a trend of lower sensitivity was observed in comparison to carbon fiber microelectrodes (CFMEs). Similar to CFMEs, the current for CNPEs is linear for dopamine from $0.1-10 \mu \mathrm{M}$ and CNPEs have a LOD of $25 \pm 5 \mathrm{nM}$. CNPEs were stable over time when the applied triangle waveform was -0.4 to $1.3 \mathrm{~V}$ and back at $400 \mathrm{~V} / \mathrm{s}$. CNPEs were used to detect exogenous and endogenous dopamine in Drosophila larvae which verified these electrodes were suitable for in vivo studies. CNPEs are advantageous for neurotransmitter measurements in distinct brain regions of adult Drosophila, which will enable studies of how genetics or behavior affects neurotransmission regulation.
\end{abstract}




\subsection{Introduction}

Carbon fiber microelectrodes (CFMEs) are traditionally used with fast-scan cyclic voltammetry (FSCV) to study rapid neurotransmitter changes in vivo. ${ }^{1}$ They offer realtime detection of catecholamines with high sensitivity and selectivity. Traditional CFMEs are $7 \mu \mathrm{m}$ in diameter ${ }^{2}$; however, smaller electrodes would be useful for neurochemical studies in small organisms such as Drosophila melanogaster (the fruit fly). Drosophila is a convenient model organism because it has homologous neurotransmitters with mammals and is easy and fast for genetic manipulation. The larval fly central nervous system is extremely small, only $100 \mu \mathrm{m}$ wide and the brain is about $8 \mathrm{~nL}$ in volume. ${ }^{3}$ CFMEs have been used to make electrochemical measurements of exogenously applied dopamine in the adult fly mushroom body. ${ }^{4}$ In addition, endogenous, stimulated dopamine changes have been measured in a single fruit fly larva. ${ }^{3}$ Drosophila have glial sheaths surrounding their neuropil that can be tough to penetrate. In larva, a cut surface is made to insert the electrode $\mathrm{e}^{3,5,6}$ and in adults, collagenase has been applied to chemically digest the tissue. ${ }^{4,7}$ Therefore, studying the release of endogenous neurotransmitters with better spatial resolution requires a small, dagger-like electrode that can penetrate through the tough glial sheath barrier with minimal tissue damage.

Over the past few decades, nanoelectrodes have been developed for electrochemical applications. Carbon electrodes are preferred for neurotransmitter applications with advantages such as low cost, wide potential window, and good adsorption properties. ${ }^{8}$ For smaller electrodes, carbon fibers can be either flame etched or electrochemically etched to sub-micron tips. ${ }^{9-12}$ Carbon nanomaterials, such as nanotubes, could also be used as smaller electrodes. Carbon nanofiber microelectrodes have been 
developed for neurotransmitter detection, but they are on a larger chip and not easily implantable. ${ }^{13}$ Small carbon paste electrodes have been made for scanning electrochemical microscopy studies, but are not easy to batch fabricate. ${ }^{14}$ Tiny, nanometer sized electrodes have been made using a single-walled carbon nanotube either sticking out ${ }^{15}$ or on a silicon wafer. ${ }^{16}$ Alternatively, some methods completely insulate an etched carbon fiber except for the very tip leaving an effective diameter of a few nanometers. ${ }^{17,18}$ However, insulation is difficult and a single carbon nanotube or $1 \mathrm{~nm}$ sized probe does not have the robustness to be implanted into tissue.

For in vivo measurements in Drosophila, we desire a sharp, carbon nanoelectrode with high sensitivity to detect nanomolar concentrations and a facile fabrication. Carbon nanopipette electrodes (CNPEs) are nanometer sized electrodes primarily used for electrophysiological measurements and delivering fluids into cells. ${ }^{19,20}$ CNPEs are fabricated by depositing a carbon layer on the inside of a pulled glass/quartz capillary. The capillary can then be etched to expose the tip. CNPEs are batch fabricated in a furnace and are rigid because of the quartz insulation. While many of the past designs have been hollow pipettes, allowing for drug delivery to cells, changing the manufacturing conditions can lead to a solid tip with a 50-400 nm diameter. Here, solidtipped CNPEs and FSCV are coupled for the first time.

The objective of this study was to characterize the electrochemical properties of CNPEs using FSCV for the detection of dopamine and test their suitability for measurements in Drosophila. CNPEs that were approximately $250 \mathrm{~nm}$ in diameter at the tip with 100-150 $\mu \mathrm{m}$ exposed carbon length were tested. The detected dopamine current was stable at CNPEs with an optimized triangular waveform of $-0.4 \mathrm{~V}$ to $1.3 \mathrm{~V}$ at a scan 
rate of $400 \mathrm{~V} / \mathrm{s}$ and a frequency of $10 \mathrm{~Hz}$. The current was linear with dopamine concentration up to $10 \mu \mathrm{M}$. CNPEs are sharp and robust enough to successfully penetrate into a Drosophila larva without breaking and dopamine could be measured in the fly. CNPEs coupled with FSCV will allow for fast, real-time measurements of dopamine in specific brain regions of the Drosophila.

\subsection{Methods}

\subsubsection{Solutions and Chemicals}

All reagents were purchased from Fisher Scientific (Fair Lawn, NJ) unless otherwise specified. Dopamine hydrochloride was purchased from Sigma-Aldrich (St. Louis, $\mathrm{MO}$ ), dissolved in $0.1 \mathrm{M} \mathrm{HClO}_{4}$ for a $10 \mathrm{mM}$ stock solution and diluted daily in phosphate buffered saline (PBS) for testing. The PBS was $131.25 \mathrm{mM} \mathrm{NaCl}, 3.0 \mathrm{mM}$ $\mathrm{KCl}, 10.0 \mathrm{mM} \mathrm{NaH}_{2} \mathrm{PO}_{4}$ monohydrate, $1.2 \mathrm{mM} \mathrm{MgCl}_{2}$ hexahydrate, $2.0 \mathrm{mM} \mathrm{Na} 2 \mathrm{SO}_{4}$ anhydrous, and $1.2 \mathrm{mM} \mathrm{CaCl}_{2}$ dihydrate with the $\mathrm{pH}$ adjusted to 7.4. Sodium chloride was purchased from VWR International LLC (West Chester, PA), sodium phosphate from Ricca Chemical Company (Arlington, TX) and calcium chloride from SigmaAldrich. All aqueous solutions were made with deionized water (Milli-Q Biocel, Millipore, Billerica, MA).

\subsubsection{Carbon Nanopipette Electrode Fabrication}

CNPEs were fabricated with $1 \mathrm{~mm}$ outer diameter, $0.7 \mathrm{~mm}$ inner diameter, filamented quartz capillaries of $10 \mathrm{~cm}$ length (Sutter Instrument Co., Novato, CA). Pipettes were pulled using a Sutter P-2000 laser-based pipette puller with the parameters: HEAT 800, FIL 4, VEL 60, DEL 128, and PULL 100. Chemical vapor deposition (CVD) was performed on the pipettes in a 3-zone horizontal tube furnace (Carbolite HVS, Hope 
Valley, UK) with a $1.3^{\prime}$ inner diameter quartz tube at $900{ }^{\circ} \mathrm{C}$, with flow conditions of 400 standard cubic centimeters per minute $(\mathrm{sccm})$ methane and $600 \mathrm{sccm}$ argon, for a 6 hour duration. During deposition, the pipette tips were oriented against the flow of the gas, i.e., the tip pointed upstream. Pipettes were cooled under argon flow to prevent the oxidation of the carbon at elevated temperatures. The carbon deposited selectively inside the pipette, not on the outer surface.

The carbon-coated pipettes were etched in 5:1 buffered hydrofluoric acid (Transene Co. Inc., Danvers, MA) for 10 minutes followed by a 10-minute rinse in deionized water. The pipettes were inspected under an optical microscope (Olympus Corp. BX-51) and imaged with a SEM (FEI Quanta 600 ESEM, Hillsboro, OR). The tip outer diameter ranged from 50 to $400 \mathrm{~nm}$ and the exposed carbon tip length depended on the etch time and tolerances of the pipette puller, but typically was between 125 and $175 \mu \mathrm{m}$ for etch times of 10 minutes. To ensure the pipettes were properly sealed, CNPEs were connected to the headstage of a HEKA EPC 10 patch-clamp amplifier using a standard 1.0mm HEKA pipette holder. The pipettes were also connected to a pressureinjection pump (Eppendorf Femtojet, Happauge, NY). The CNPE tip was submerged in phosphate buffered saline (HyClone, PBS1X) and a silver chloride wire was used as a counter/reference electrode in solution in a 2-electrode configuration. The digital lock-in module of the PATCHMASTER software was used to measure the equivalent capacitance of the CNPE interface with a $10 \mathrm{mV}, 1 \mathrm{kHz}$ sinusoidal potential, as the pressure within the pipette was adjusted between 0 and $300 \mathrm{kPa}$. The tip was first checked for bubbles, which would indicate a non-sealed or broken tip, and then the capacitance was monitored with changing pressure. The capacitance is proportional to the 
electrode interfacial area, and if it is stable with varying pressure it indicates that there is minimal capillary rise and that the tip is well-sealed. CNPEs that were not well sealed were discarded.

\subsubsection{Scanning Electron Microscopy}

Scanning electron microscopy was performed in a FEI Quanta 600 ESEM (FEI, Hillsboro, Oregon) in secondary electron mode. CNPEs were adhered to a standard sample mount with carbon tape such that the CNPE axis was orthogonal to the electron beam. A short working distance $(5 \mathrm{~mm})$ and low accelerating voltage $(2 \mathrm{keV})$ were used in high-vacuum mode to attain enhanced surface detail and to minimize charging effects. $^{21}$ The Environmental SEM provides a large sample chamber that allows CNPEs to be mounted without breaking or modifying them.

\subsubsection{Instrumentation and Electrochemistry}

Fast-scan cyclic voltammograms were collected using a Chem-Clamp potentiostat (Dagan, Minneapolis, MN). TarHeel CV software (gift of Mark Wightman, University of North Carolina) was used for data collection and analysis. The hardware and data acquisition were the same as previously described. ${ }^{22}$ A triangular waveform was applied to the electrode. The electrode was scanned at a scan rate of $400 \mathrm{~V} / \mathrm{s}$ from $-0.4 \mathrm{~V}$ to $1.3 \mathrm{~V}$ and back at a frequency of $10 \mathrm{~Hz}$ unless otherwise noted. $\mathrm{A} \mathrm{Ag} / \mathrm{AgCl}$ wire was used as a reference electrode. The flow injection apparatus with a six-port, stainless steel HPLC loop injector used is the same as previously described. ${ }^{23}$ Electrodes were tested with a $5 \mathrm{~s}$ injection time. Because carbon is deposited on the entire length of the CNPEs, a direct electrical connection was made with a silver wire in the Universal Pipette Holder (HB180, Dagan Corp., Minneapolis, MN). No backfill solution was used. The holder was connected to a $1 \mathrm{M} \Omega$ headstage (Dagan Corp., Minneapolis, MN). 


\subsubsection{Statistics}

Statistics were performed using GraphPad Prism 6.0 (GraphPad Software, San Diego, CA). Data are reported as the mean \pm standard error of the mean (SEM) for $n$ number of different samples. Significance was determined by unpaired $t$-tests and defined as $p \leq 0.05$.

\subsubsection{Detection of Exogenously Applied Dopamine in Drosophila}

Measurements of exogenously applied dopamine in Drosophila were made in isolated Drosophila ventral nerve cords. ${ }^{24}$ Third instar, wild-type Canton-S larva were collected and their ventral nerve cords were isolated and kept in PBS buffer supplemented with $11.1 \mathrm{mM}$ glucose and $5.3 \mathrm{mM}$ trehalose. Both the proximal and distal ends of the nerve cord were severed to facilitate insertion of the electrode and picospritzer pipette from opposite ends of the tissue. Picospritzing pipettes were fabricated and calibrated as previously described. ${ }^{24}$ Pipettes were backfilled with $50 \mu \mathrm{M}$ dopamine and inserted with a micromanipulator into the neuropil $20 \mu \mathrm{m}$ away from the recording electrode. The electrode was allowed to equilibrate for 10 minutes prior to data collection. A baseline recording was taken for 10 seconds prior to dopamine ejection.

\subsubsection{Endogenous Dopamine Evoked by Chrimson Channelrhodopsin Stimulation}

Virgin females with UAS-Chrimson inserted in attp18 ${ }^{25}$ (a gift of Vivek Jayaraman) were crossed with th-GAL4 (a gift of Jay Hirsh). Resulting heterozygous larvae were shielded from light and raised on standard cornmeal food mixed 250:1 with $100 \mathrm{mM}$ all-trans-retinal. A small amount of moistened Red Star yeast (Red Star, Wilwaukii, WI) was placed on top of the food to promote egg laying. The central nervous system of a third instar wandering larva was dissected in the buffer. Isolated 
ventral nerve cords were prepared and recorded from as previously described. ${ }^{26}$ The electrode was allowed to equilibrate in the tissue for 15 minutes prior to data collection. A baseline recording was taken for 10 seconds prior to stimulation. Red-orange light from a 617 nm fiber-coupled high-power LED with a $200 \mu \mathrm{m}$ core optical cable (ThorLabs, Newton, NJ) was used to stimulate the Chrimson ion channel. The light was modulated with Transistor-Transistor Logic (TTL) inputs to a T-cube LED controller (ThorLabs, Newton, NJ), which was connected to the breakout box. TTL input was driven by electrical pulses controlled by the TarHeel CV software.

\subsection{Results and Discussion}

The first goal of this study was to electrochemically characterize CNPEs using FSCV. FSCV allows measurements of rapid changes in neurotransmitter concentrations. CNPEs have traditionally been manufactured with open tips ${ }^{19}$, but that is not suitable for electrochemistry as sample would wick up into the pipette. Here, the fabrication was modified slightly to grow enough carbon to make an electrode with a solid tip 200 nm in diameter. The second goal was to test the suitability of CNPEs for dopamine measurements in Drosophila larvae, which have a very small central nervous system. This nanoscale electrode would allow for high spatial resolution measurements.

\subsubsection{Fabrication of Carbon Nanopipette Electrodes}

The carbon nanopipette electrode (CNPE) consists of a pulled-glass/quartz pipette coated with a layer of pyrolytic carbon along its entire inner surface to a thickness sufficient to seal the pipette's narrow opening (Figure 2.1). Figure 2.1A shows the fabrication process. First a quartz capillary is pulled into a fine-tip micropipette (Figure 2.1A (i)). Next, carbon is deposited by CVD until the tip is sealed with carbon (Figure 2.1A (ii)). Further up, the pipette is still hollow, which facilitates electrical 
connection via contact with a wire. Subsequent to the carbon deposition, the quartz/glass at the tip of the CNPEs is etched in buffered hydrofluoric acid to expose a desired length of a tapered carbon cylinder, typically ranging in length from 100-150 $\mu \mathrm{m}$ (Figure 2.1A (iii)). The exposed length is controlled by the etching time. For the pipette geometry used here, this corresponds to exposed length of $10-15 \mu \mathrm{m} /$ minute as measured with an optical microscope and confirmed with SEM. Figure 2.1B shows an example CNPE tip with $\sim 170 \mu \mathrm{m}$ exposed carbon and the interface with the quartz insulation. Figure $2.1 \mathrm{C}$ is an enlarged image of the quartz/carbon interface. The tip diameter of the individual CNPEs used in this work was measured with SEM and had a typical range of tip sizes 50-400 nm (Figure 2.1D). Since the glass/quartz template controls the outer dimensions of the deposited carbon, it is likely that the primary source of tip variability stems from the pipette puller parameters. Even with the variability, this fabrication method consistently yields sub-micron sized tips, an order of magnitude smaller than traditional CFMEs.

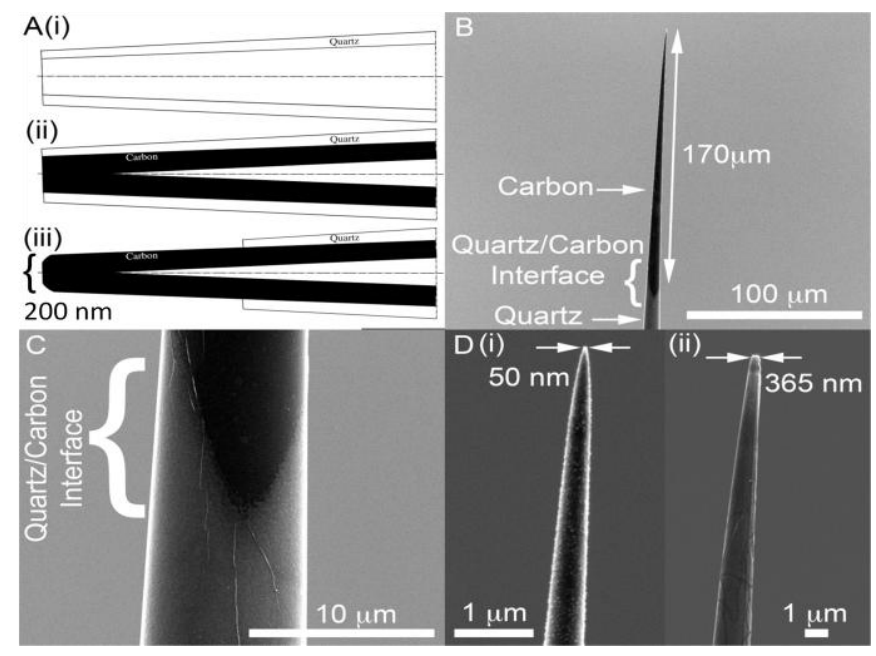

Figure 2.1. Carbon Nanopipette Electrodes. A) A schematic of the CNPE fabrication process. i) Quartz/glass pipette is pulled to form a template. ii) The pulled pipette is placed in a furnace in the presence of precursor hydrocarbons and carbon is deposited selectively along the pipette's interior surface for a sufficient amount of time until the tip is sealed with carbon. iii) The glass/quartz at the tip is wet-etched away to expose a desired length of the underlying carbon. B) SEM image of the CNPE tip profile. C) Enhanced SEM view of the quartz/carbon interface. D) Enhanced SEM view of the CNPE tips. i) Tip diameter $50 \mathrm{~nm}$. ii) Tip diameter $365 \mathrm{~nm}$ (same CNPE as in B and C). The tip and edges appear brighter due to SEM charging effects. 


\subsubsection{Comparison of CNPEs and CFMEs}

Dopamine was chosen to analyze with CNPEs because it is easily oxidized, adsorbs to carbon surfaces ${ }^{27}$, and is an important neurotransmitter. Dopamine plays a role in reward, addiction, and motor behaviors. ${ }^{28}$ Figure 2.2 shows example background subtracted cyclic voltammograms (A and B) as well as normalized current vs time plots (C and D) for dopamine at two different waveforms for a CNPE and a CFME. The first waveform (referred to as the $1.0 \mathrm{~V}$ waveform) scanned from -0.4 to $1.0 \mathrm{~V}$ at a scan rate of $400 \mathrm{~V} / \mathrm{s}$ and a frequency of $10 \mathrm{~Hz}$. The second waveform (referred to as the $1.3 \mathrm{~V}$ waveform) was the same except the switching potential was $1.3 \mathrm{~V}$. Figures $2.2 \mathrm{~B}$ and D show larger currents for both CNPEs and CFMEs with the $1.3 \mathrm{~V}$ potential limit, as expected due to oxidation of carbon. ${ }^{29}$ The CFME had more current for dopamine than the CNPE for both waveforms; however, the peak oxidation voltage is lower for CNPEs (Figures 2.2A and B). CNPEs and CFMEs have more similar time responses at the $1.3 \mathrm{~V}$ waveform than the $1.0 \mathrm{~V}$ waveform (Figures $2.2 \mathrm{C}$ and $\mathrm{D})$.

A.

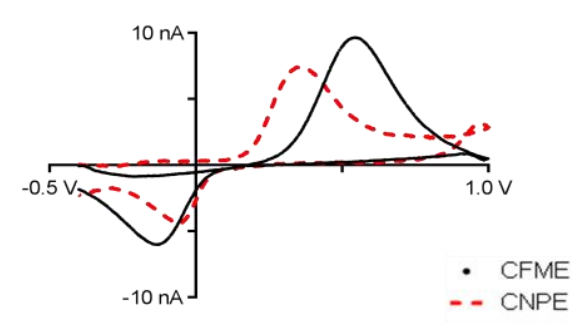

C.

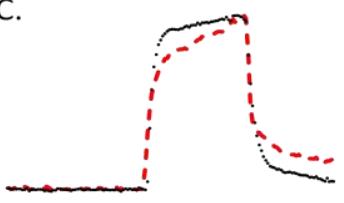

B.

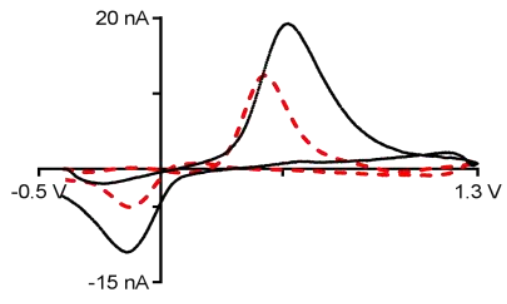

D.

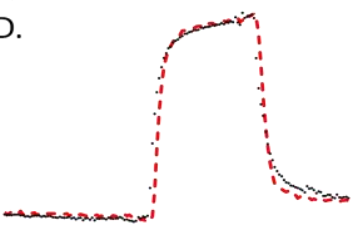

Figure 2.2. Example data for a $150 \mu \mathrm{m}$ long CNPE (red dashed line) and a $50 \mu \mathrm{m}$ long CFME (black line) with the $1.0 \mathrm{~V}$ and $1.3 \mathrm{~V}$ waveforms. Background subtracted cyclic voltammograms for $1 \mu \mathrm{M}$ dopamine are shown for (A) the $1.0 \mathrm{~V}$ waveform and (B) the $1.3 \mathrm{~V}$ waveform. Normalized current versus time plots at peak oxidation voltage for (C) the $1.0 \mathrm{~V}$ and (D) $1.3 \mathrm{~V}$ waveforms. 


\section{Table 2.1. Average electrochemical parameters for $1 \mu \mathrm{M}$ dopamine detection for CNPEs and CFMEs at $1.0 \mathrm{~V}$ and $1.3 \mathrm{~V}$ waveforms.}

$\begin{array}{lccc} & i_{p, a}(\mathrm{nA}) & \text { Background current }(\mathrm{nA}) & \Delta \mathrm{E}_{\mathrm{P}}(\mathrm{V}) \\ \text { CFME 1.0V }^{a} & 11 \pm 2 & 330 \pm 70 & 0.69 \pm 0.02 \\ \text { CNPE 1.0V } & 7.4 \pm 2 & 490 \pm 110 & 0.50 \pm 0.02^{d} \\ \text { CFME 1.3V } & 19 \pm 2 & 410 \pm 80 & 0.66 \pm 0.01 \\ \text { CNPE 1.3V } & 14 \pm 3 & 570 \pm 160 & 0.52 \pm 0.01^{a}\end{array}$

$i_{p, a}$ is peak oxidative current; $\Delta \mathrm{E}_{\mathrm{p}}$ is the difference between the oxidative and reductive potentials. ${ }^{a} n=6 .{ }^{b} n=8 .{ }^{c} n=11 .{ }^{d}$ Data are significantly different than CFMEs at the same waveform $(p<0.0001)$.

Table 2.1 shows average peak oxidative currents $\left(i_{p, a}\right)$, background currents, and $\Delta \mathrm{E}_{\mathrm{P}}$ for CNPEs $(150 \mu \mathrm{m}$ in length, $\sim 250 \mathrm{~nm}$ diameter) and CFMEs $(50-75 \mu \mathrm{m}$ in length, $7 \mu \mathrm{m}$ diameter) at the $1.0 \mathrm{~V}$ and $1.3 \mathrm{~V}$ waveforms. The average peak oxidative current $\left(i_{p, a}\right)$ for $1 \mu \mathrm{M}$ dopamine is about $30 \%$ lower for CNPEs than CFMEs at both waveforms; however, the difference was not significant (unpaired t-test, $1.0 \mathrm{~V}$ waveform, $\mathrm{p}=0.1273$; 1.3 V waveform, $\mathrm{p}=0.2353$ ). The background currents were compared using the maximum value for the forward scan. Background currents for CNPEs were higher than CFMEs, although not significantly (unpaired t-test, $1.0 \mathrm{~V}$ waveform, $\mathrm{p}=0.2484 ; 1.3 \mathrm{~V}$ waveform, $\mathrm{p}=0.3730)$. A lower oxidation current correlated with lower background charging current was expected; however, this was not true for these CNPEs. Background current is proportional to surface area. The calculated surface area for the CNPEs is actually higher $(\sim 1.5 \mathrm{x})$ than the CFMEs. Note the exposed length of the CNPEs is about 3 times the length of an average carbon fiber. The capacitive currents for about $100 \mu \mathrm{m}$ long CNPEs were $490 \mathrm{pF} \pm 200(n=20)$ and this would translate to surface areas of about $860 \mu \mathrm{m}^{2}$, which is more on the order of magnitude of CFMEs. The larger background current of CNPEs correlates to higher capacitance which is proportional to noise.

Therefore, one future goal is to decrease the exposed carbon length. 
$\Delta \mathrm{E}_{\mathrm{p}}$ is the difference between the oxidative and reductive peak potentials. The average $\triangle \mathrm{E}_{\mathrm{p}}$ for CNPEs is significantly lower than CFMEs for the two waveforms (Table 1, unpaired t-test $\mathrm{p}<0.0001)$. There is no significant difference in $\triangle \mathrm{E}_{\mathrm{p}}$ for CNPEs for the $1.0 \mathrm{~V}$ and $1.3 \mathrm{~V}$ waveforms $(\mathrm{p}=0.4010)$. The decrease in $\triangle \mathrm{E}_{\mathrm{p}}$ for CNPEs compared to CFMEs implies reduced overpotential for dopamine. This reduction, along with the slightly lower sensitivity, likely means the carbon type is different between electrodes. The CNPE carbon is amorphous with graphitic islands, and has surface functional groups that depend on deposition conditions. ${ }^{30}$ The lower $\Delta \mathrm{E}_{\mathrm{p}}$ denotes faster electron transfer kinetics likely modulated by functional groups on the CNPEs or differences in the amount of edge plane graphite sites, which play a role in electron transfer and adsorption reactivity. $^{8}$

\subsubsection{CNPE Stability Over Time}

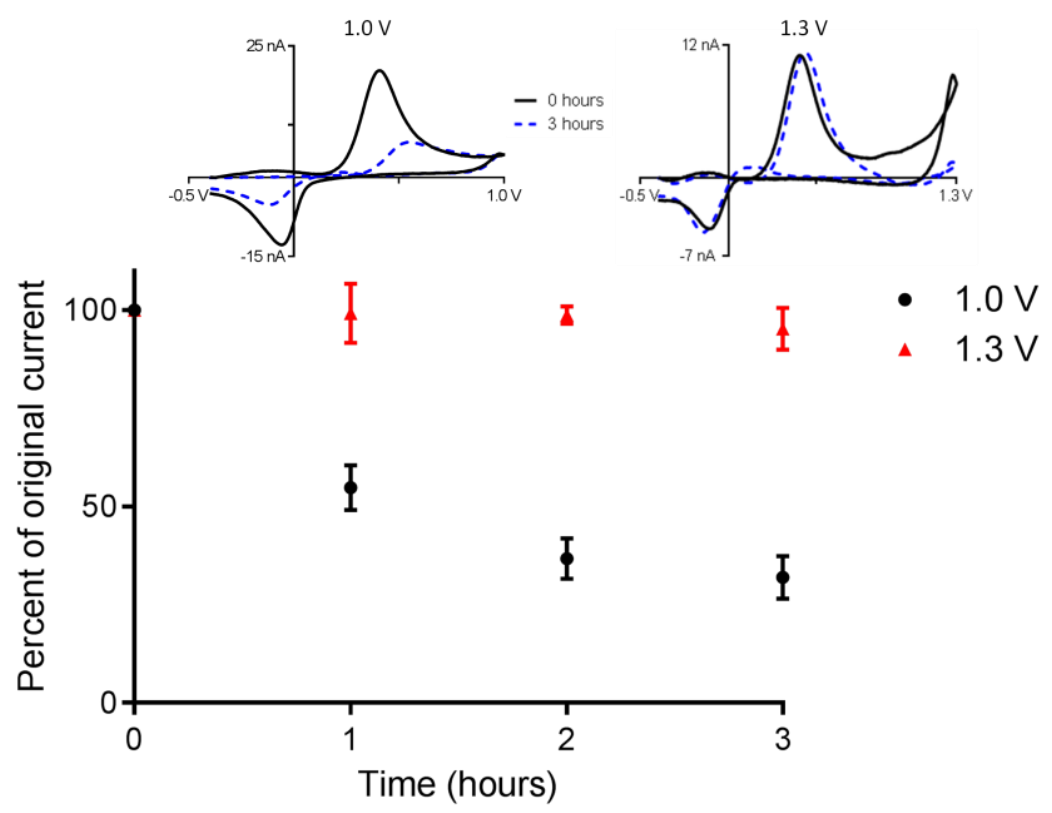

Figure 2.3. Stability of CNPEs over three hours at the $1.0 \mathrm{~V}$ waveform (black circles) and $1.3 \mathrm{~V}$ waveform (red triangles). Insets show example cyclic voltammograms for both waveforms at initial measurements and after three hours. 
Electrode stability is important for in vivo experiments which can last hours. ${ }^{31}$ To test stability, the $1.0 \mathrm{~V}$ waveform was applied continuously to the CNPE in buffer and the response to a five-second injection of $1 \mu \mathrm{M}$ dopamine was measured every hour. Current was normalized for each electrode to the first response to dopamine to take into account differences in individual electrodes. Figure 2.3 shows at the $1.0 \mathrm{~V}$ waveform, the CNPE sensitivity dropped to $32 \%$ of the original current after 3 hours. The $1.0 \mathrm{~V}$ inset shows example cyclic voltammograms taken at the first injection of dopamine and after three hours. The oxidative and reductive peak voltages shifted outward, signifying slower electron transfer kinetics accompanied the decrease in sensitivity. We hypothesize the surface of the electrode is fouled which would reduce the sensitivity and slow the transfer kinetics; however, dopamine diffuses to the electrode and some current is still measured from electron tunneling.

This problem of electrode surface fouling is overcome by electrochemically renewing the surface. Scanning to $1.3 \mathrm{~V}$ allows for the regeneration of a fresh carbon surface and maintains electrode sensitivity. ${ }^{29}$ For the stability experiments using the $1.3 \mathrm{~V}$ waveform, the CNPEs were allowed to stabilize with the waveform applied for 30 minutes before taking the initial measurement due to the oxidation of the electrode surface. ${ }^{22}$ If not allowed to stabilize, the signal actually increases during this time due to increased surface area from carbon-carbon bonds breaking and increased adsorption due to carbon functional groups. The peak oxidative current was constant over three hours (Figure 2.3) indicating that CNPEs are stable at this waveform and suitable for longer in vivo studies. This is confirmed by the inset CVs which show the sensitivity and the electron transfer kinetics remained the same. From this stability experiment, we 
determined the $1.3 \mathrm{~V}$ waveform is optimal and used this waveform for the remaining studies.

\subsubsection{CNPE Characterization}
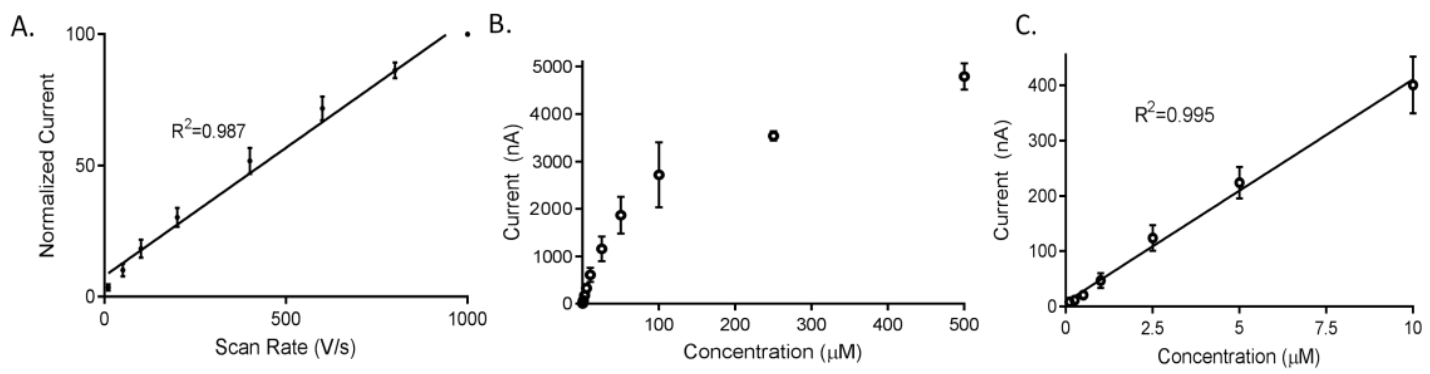

Figure 2.4. Characterization of CNPEs. (A) Normalized peak oxidative current for $1 \mu \mathrm{M}$ dopamine vs scan rate. The plot is linear for CNPE $(n=4)$ showing the kinetics are adsorption controlled. (B) Peak oxidative current vs concentration $(n=5)$. (C) CNPEs show a linear response in current up to $10 \mu \mathrm{M}$.

Figure 2.4 shows that peak oxidation current for $1 \mu \mathrm{M}$ dopamine is proportional to scan rate at CNPEs $\left(\mathrm{R}^{2}=0.987, n=4\right)$. Frequency was varied to keep equal time in between scans. Current is normalized to the maximum value per electrode to minimize effects due to varying sensitivity of different electrodes. This plot indicates that the kinetics are more adsorption controlled than diffusion controlled, similar to other carbon microelectrodes. ${ }^{27}$ Figure 2.4B shows peak currents for various dopamine concentrations $(100 \mathrm{nM}$ to $500 \mu \mathrm{M})$. Current is linear with concentration up to $10 \mu \mathrm{M}$ (Figure $2.4 \mathrm{C}$ ), but deviates from linearity at high concentrations, which indicates the kinetics are no longer adsorption controlled at high concentrations. The adsorption sites become occupied and diffusion to the electrode surface limits the reaction. The average LOD, calculated from the $100 \mathrm{nM}$ data, was $27 \mathrm{nM} \pm 4 \mathrm{nM}(n=5)$. 


\subsubsection{Measurements of Exogenous Dopamine in Drosophila}

A.

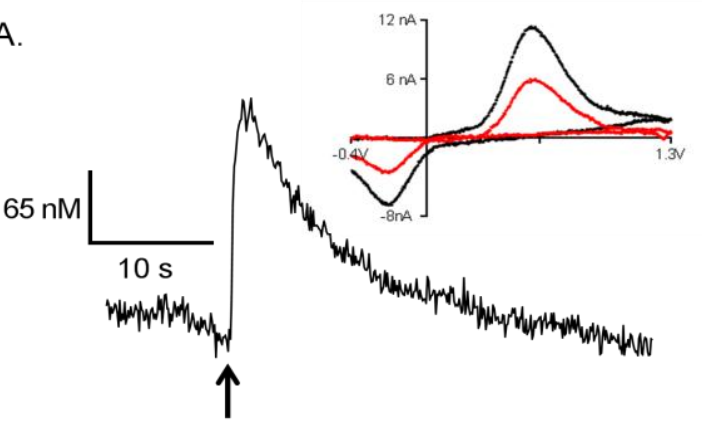

B.

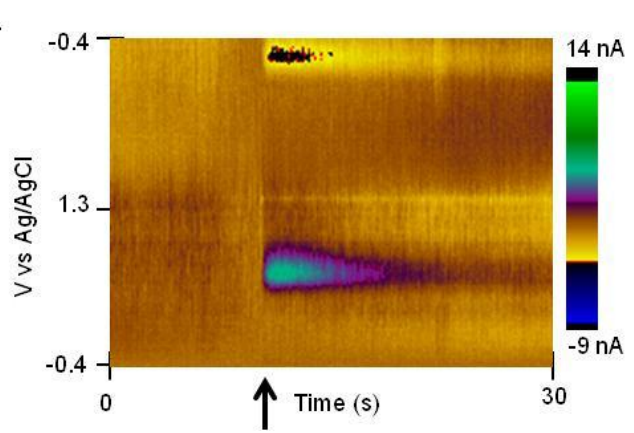

Figure 2.5. CNPE measurements of exogenously applied dopamine in Drosophila using the $1.3 \mathrm{~V}$ waveform. (A) $1.8 \mathrm{~nL}$ of a $50 \mu \mathrm{M}$ dopamine solution was applied (arrow) into the tissue and $190 \mathrm{nM}$ detected. (Inset) Background subtracted cyclic voltammograms. The red line is exogenously applied dopamine in a larva CNS. The black line shows applied dopamine outside the CNS in buffer. (B) Color plot showing applied dopamine (arrow) in a Drosophila larva. The green area is the oxidation peak and the dark blue is the reduction peak for dopamine.

Exogenous dopamine was applied similarly to that of previous studies. ${ }^{24} \mathrm{~A} \mathrm{CNPE}$ was implanted into wild-type Canton-S isolated Drosophila larval ventral nerve cords. A micropipette with $50 \mu \mathrm{M}$ dopamine was implanted $20 \mu \mathrm{m}$ from the CNPE. Figure 2.5A shows an example current versus time plot for an application of $1.8 \mathrm{~nL}$ of $50 \mu \mathrm{M}$ dopamine (arrow). At the peak, $190 \mathrm{nM}$ dopamine was detected. Dopamine is cleared from the extracellular space by dopamine $\operatorname{transporters}^{6}$ and the elevated dopamine concentration lasts only about 20 seconds. The inset red cyclic voltammogram shows detected dopamine in the larval CNS and the black cyclic voltammogram is when the same amount of dopamine was applied in buffer, not a tissue sample. The CVs look the same, indicating the electrode can measure dopamine in tissue. The current measured in the fly is lower than in buffer because uptake occurs in tissue and dopamine can also diffuse away. Figure 2.5B shows data in a three-dimensional false-color plot for exogenously applied dopamine in a larva. Consecutive voltammograms are plotted over time on the x-axis. The $y$-axis is applied voltage and current is shown in false color. 
Green is dopamine oxidation and blue/yellow is dopamine reduction. The positions of the oxidation and reduction peaks verify dopamine is detected in vivo.

\subsubsection{Measurements of Endogenous Dopamine in Drosophila Evoked by Chrimson Channelrhodopsin Stimulation}

To test the use of CNPEs to detect endogenous dopamine, dopamine release was stimulated with a light sensitive cation channel called Chrimson. Chrimson is a channelrhodopsin that is more red-shifted than the traditional channelrhodopsin $2 .{ }^{25}$ Upon light stimulation, the channel opens and sodium ions can enter, depolarizing the neuron and causing an action potential. Th-GAL4 flies were genetically modified with UASChrimson so Chrimson is expressed in Drosophila dopaminergic motor neurons. ${ }^{25}$ The CNPE was inserted in an isolated ventral nerve cord and the $1.3 \mathrm{~V}$ waveform was applied. Figure 2.6 shows current measured during a 5 s continuous stimulation with red light. Figure 2.6A shows the evoked dopamine signal rises quickly upon stimulation and falls after the light is turned off. This is consistent with dopamine release and clearance. The concentration versus time plot is made using an in vitro calibration to convert maximum peak oxidation current to concentration of dopamine. The cyclic voltammogram (Figure 2.6B) verifies dopamine was detected. This process was repeated and dopamine $(630 \pm 20 \mathrm{nM})$ was detected for three separate larvae verifying CNPEs are suitable for in vivo dopamine measurements. The CNPE was a bit noisy for this application, as the full electrode might not have been placed in the tissue. Reducing the exposed length should solve that problem and future experiments will optimize implantation. 
A.

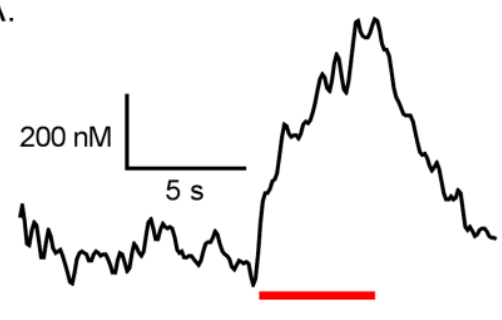

B.

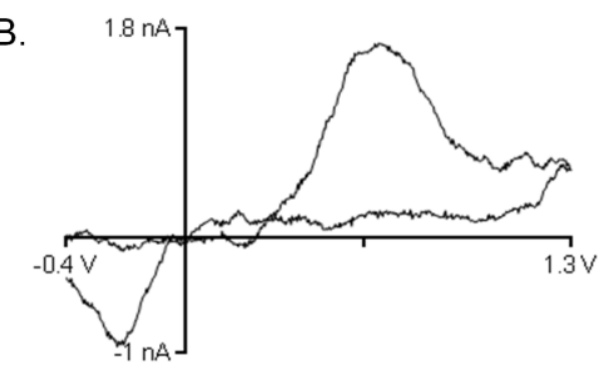

Figure 2.6. Example CNPE measurements of endogenous dopamine evoked by a 5 second continuous red light stimulation.

The batch fabrication of robust, nanosized electrodes suitable for in vivo studies is difficult and most methods to fabricate smaller electrodes have involved etching a single electrode by hand. The CNPEs developed here are batch fabricated and have the robustness to be implanted for in vivo studies. The sensitivity of CNPEs with FSCV is comparable to traditional CFMEs, maybe slightly less for a given exposed length because of the smaller area, but CNPEs are able to measure endogenous dopamine in Drosophila larvae. In the future, the exposed carbon length needs to be optimized to minimize noise while maintaining desired sensitivity. Efforts are being made to increase batch yield and maintain reproducibility between CNPEs. For high spatial resolution measurements in Drosophila, the length should be reduced as much as feasible. Mechanically polishing CNPEs to disk electrodes could be a future challenge and would allow for measurements from discrete regions and at single neuronal cells. The ability to measure endogenous dopamine release in Drosophila will allow for studies on how genetics or behavior affects neurotransmission regulation. CNPEs could also be applied to study other neurotransmitters such as serotonin and octopamine in the future. 


\subsection{Conclusions}

We fabricated solid-tipped CNPEs which provide high spatially resolved measurements of dopamine. CNPEs were characterized for the first time with FSCV and their electrochemical signals for dopamine were suitable for in vivo measurements in Drosophila. When compared to CFMEs, CNPEs are not quite as sensitive per unit area but show faster electron transfer kinetics. CNPEs are stable with the $1.3 \mathrm{~V}$ waveform and sensitive enough to detect evoked dopamine release in Drosophila larvae. Coupled with FSCV, CNPEs could be used to measure real-time dopamine changes in the adult fly.

Future studies in specific brain regions will give a better understanding of neurotransmission underlying discrete physiological processes. 


\subsection{References}

1. Robinson, D. L.; Hermans, A.; Seipel, A. T.; Wightman, R. M. Monitoring Rapid Chemical Communication in the Brain. Chem. Rev. 2008, 108 (7), 25542584.

2. Chadchankar, H.; Yavich, L. Characterization of a $32 \mathrm{~mm}$ diameter carbon fiber electrode for in vivo fast-scan cyclic voltammetry. Journal of Neuroscience Methods 2011, 211 (2), 218-226.

3. Vickrey, T. L.; Condron, B.; Venton, B. J. Detection of Endogenous Dopamine Changes in Drosophila melanogaster Using Fast-Scan Cyclic Voltammetry. Analytical Chemistry 2009, 81, 9306-9313.

4. Makos, M.; Kim, Y.; Han, K.; Heien, M. L.; Ewing, A. In vivo electrochemical measurements of exogenously applied dopamine in Drosophila melanogaster. Analytical Chemistry 2009, 81 (5), 1848-1854.

5. Xiao, N.; Venton, B. J. Rapid, Sensitive Detection of Neurotransmitters at Microelectrodes Modified with Self-assembled SWCNT Forests. Analytical Chemistry 2012, 84, 7816-7822.

6. Vickrey, T. L.; Venton, B. J. Drosophila Dopamine2-like Receptors Function as Autoreceptors. ACS Chemical Neuroscience 2011, 2, 723-729.

7. Makos, M.; Han, K.; Heien, M. L.; Ewing, A. Using in Vivo Electrochemistry to Study the Physiological Effects of Cocaine and Other Stimulants on the Drosophila melanogaster Dopamine Transporter. ACS Chemical Neuroscience 2010, 1 (1), 74-83.

8. McCreery, R. L. Advanced Carbon Electrode Materials for Molecular Electrochemistry. Chem. Rev. 2008, 108, 2646-2687.

9. Huang, W.; Pang, D.; Tong, H.; Wang, Z.; Cheng, J. A Method for the Fabrication of Low-Noise Carbon Fiber Nanoelectrodes. Analytical Chemistry 2001, 73, 1048-1052.

10. Strand, A. M.; Venton, B. J. Flame Etching Enhances the Sensitivity of CarbonFiber Microelectrodes. Analytical Chemistry 2008, 80, 3708-3715.

11. Kawagoe, K. T.; Jankowski, J. A.; Wightman, R. M. Etched Carbon-Fiber Electrodes as Amperometric Detectors of Catecholamine Secretion from Isolated Biological Cells. Analytical Chemistry 1991, 63, 1589-1594.

12. Strein, T. G.; Ewing, A. Characterization of Submicron-Sized Carbon Electrodes Insulated with a Phenol-Allylphenol Copolymer. Analytical Chemistry 1992, 64, 1368-1373. 
13. Koehne, J.; Marsh, M.; Boakye, A.; Douglas, B.; Kim, I.; Chang, S.-Y.; Jang, D.P.; Bennet, K.; Kimble, C.; Andrews, R.; Meyyappan, M.; Lee, K. H. Carbon nanofiber electrode array for electrochemical detection of dopamine using fast scan cyclic voltammetry. Analyst 2011, 136 (9), 1802-1805.

14. Satpati, A. K.; Bard, A. J. Preparation and Characterization of Carbon Powder Paste Ultramicroelectrodes as Tips for Scanning Electrochemical Microscopy Applications. Analytical Chemistry 2012, 84, 9498-9504.

15. Campbell, J. K.; Sun, L.; Crooks, R. M. Electrochemistry Using Single Carbon Nanotubes. J. Am. Chem. Soc. 1999, 121, 3779-3780.

16. Heller, I.; Kong, J.; Heering, H. A.; Williams, K. A.; Lemay, S. G.; Dekker, C. Individual Single-Walled Carbon Nanotubes as Nanoelectrodes for Electrochemistry. Nano Letters 2005, 5 (1), 137-142.

17. Chen, S.; Kucernak, A. Fabrication of carbon microelectrodes with an effective radius of $1 \mathrm{~nm}$. Electrochemistry Communications 2002, 4, 80-85.

18. Wang, C.; Chen, Y.; Wang, F.; Hu, X. Fabrication of nanometer-sized carbon electrodes by the controllable electrochemical deposition. Electrochimica Acta 2005, 50, 5588-5593.

19. Schrlau, M. G.; Falls, E. M.; Ziober, B. L.; Bau, H. H. Carbon nanopipettes for cell probes and intracellular injection. Nanotechnology 2008, 19 (1).

20. Schrlau, M. G.; Dun, N. J.; Bau, H. H. Cell Electrophysiology with Carbon Nanopipettes. ACS NANO 2009, 3 (3), 563-568.

21. Joy, D. C.; Joy, C. S. Low voltage scanning electron microscopy. Micron 1996, 27 (3-4), 247-263.

22. Heien, M. L.; Phillips, P. E.; Stuber, G. D.; Seipel, A. T.; Wightman, R. M. Overoxidation of carbon-fiber microelectrodes enhances dopamine adsorption and increases sensitivity. Analyst 2003, 128, 1413-1419.

23. Huffman, M. L.; Venton, B. J. Electrochemical Properties of Different CarbonFiber Microelectrodes Using Fast-Scan Cyclic Voltammetry. Electroanalysis 2008, 20 (22), 2422-2428.

24. Vickrey, T. L.; Xiao, N.; Venton, B. J. Kinetics of the dopamine transporter in Drosophila larva. ACS Chemical Neuroscience 2013, 4 (5), 832-837.

25. Klapoetke, N. C.; Murata, Y.; Kim, S. S.; Pulver, S. R.; Birdsey-Benson, A.; Cho, Y. K.; Morimoto, T. K.; Chuong, A. S.; Carpenter, E. J.; Tian, Z.; Wang, J.; Xie, Y.; Yan, Z.; Zhang, Y.; Chow, B. Y.; Surek, B.; Melkonian, M.; Jayaraman, V.; Constantine-Paton, M.; Wong, G. K.-S.; Boyden, E. S. 
Independent optical excitation of distinct neural populations. Nature Methods 2014, 11, 338-346.

26. Borue, X.; Cooper, S.; Hirsh, J. Quantitative evaluation of serotonin release and clearance in Drosophila. J. Neuroscience 2009, 179 (2), 300-308.

27. Bath, B. D.; Michael, D. J.; Venton, B. J.; Joseph, J. D.; Runnels, P. L.; Wightman, R. M. Subsecond Adsorption and Desorption of Dopamine at Carbon-Fiber Microelectrodes. Analytical Chemistry 2000, 72, 5994-6002.

28. Arbuthnott, G. W.; Wickens, J. Space, time and dopamine. Trends in Neurosciences 2007, 30, 62.

29. Takmakov, P.; Zachek, M. K.; Keithley, R. B.; Walsh, P. L.; Donley, C.; McCarty, G. S.; Wightman, R. M. Carbon Microelectrodes with a Renewable Surface. Analytical Chemistry 2010, 82, 2020-2028.

30. Vitol, E. A.; Schrlau, M. G.; Bhattacharyya, S.; Ducheyne, P.; Bau, H. H.; Friedman, G.; Gogotsi, Y. Effects of Deposition Conditions on the Structure and Chemical Properties of Carbon Nanopipettes. Chemical Vapor Deposition 2009, 15, 204-208.

31. Nguyen, M.; Lee, S.; Ross, A.; Ryals, M.; Choudhry, V.; Venton, B. J. Characterization of Spontaneous, Transient Adenosine Release in the Caudate-Putamen and Prefrontal Cortex. PLoS ONE 2014, 9 (1). 


\section{Chapter 3}

\section{Conclusions and Future Directions}


In this thesis, I have characterized a modified CNPE that is an order of magnitude smaller at the tip than traditional CFMEs. Even with this nanosized tip, dopamine measurements are comparable to CFMEs. In this chapter I present two possible improvements to CNPEs as well as novel biological applications.

\subsection{Electrocoating CNPEs with Nafion}

CNPEs are advantageous because the nanometer size tip allows for high spatial resolution measurements. Furthermore, CNPEs have good sensitivity as well as fast electron transfer kinetics. There are also a few disadvantages of working with such a small electrode. The end of a CFME etches away if left at a potential of $1.3 \mathrm{~V}$ or higher for an extended amount of time. ${ }^{1}$ In chapter 2, CNPEs proved to be stable for three hours; however, I hypothesize the tip of a CNPE also etches some due to its nanometer-sized diameter. This is not normally an issue unless behavioral studies are done over a period of days, but with nanometer sized tips, they might break after a shorter period of time. To reduce etching of the carbon surface, I propose electrocoating with Nafion. Coating the surface of CNPEs with Nafion would serve three purposes. If the CNPE carbon surface does become etched over time at a switching potential of $1.3 \mathrm{~V}$, coating with Nafion could possibly reduce etching and keep the carbon surface stable. Second, electrocoating CFMEs with Nafion has shown to improve selectivity for electroactive neurotransmitters such as dopamine. ${ }^{2}$ Nafion is highly conductive to cations, so the goal of this modification would be to increase the sensitivity of CNPEs for dopamine which is positively charged at physiological $\mathrm{pH}$. Lastly, coating CFMEs with Nafion has shown to reduce electrode surface fouling while increasing sensitivity for serotonin. ${ }^{3}$ Serotonin and octopamine are two neurotransmitters of interest in Drosophila, so reducing fouling and 
increasing sensitivity with Nafion is important for future studies. In a past study with CFMEs, the Nafion film thickness was $\sim 300 \mathrm{~nm} .{ }^{3}$ With this coating, CNPEs would still be smaller than CFMEs at the tip.

\subsection{Implementation of a Catalyst for Selective CVD}

The carbon layer that extends the length of the CNPE is advantageous for easy, direct electrical connections. However, with the longer chemical vapor deposition times to form a solid tip, there is the possibility of carbon depositing on the outer surface of the pipette. This would cause a layer of extra carbon that extends up the pipette for a greater exposed carbon area. The increased area could result in larger capacitance which results in increased noise. ${ }^{4}$ Carbon depositing on the outer surface of the pipette could also cause low reproducibility between CNPEs. The original fabrication of carbon nanopipettes utilized a ferric nitrate catalyst solution deposited only on the inner surface of the pipette. ${ }^{5,6}$ Adding this catalyst solution back into the fabrication method might facilitate selective carbon deposition and possibly result in more reproducible batch fabrication of CNPEs. Using a catalyst may also decrease carbon deposition time.

\subsection{Direct Measurements from Drosophila Mushroom Bodies}

Dopamine neurons are found in wide areas of the brain including the mushroom bodies $^{7}$, and mushroom bodies in Drosophila are known to be involved in learning and memory. ${ }^{8}$ One possible application of CNPEs is to make real-time measurements of dopamine from different areas of the mushroom bodies. Each region may only be 10's of micrometers, so the high spatial resolution of CNPEs would make these measurements possible. PAM, a cluster of dopamine neurons, projects to the medial portion of the 
horizontal lobes of the mushroom bodies. ${ }^{9}$ With CNPEs we could study dopamine release and uptake in this area of the mushroom body and carry out experiments to explore its possible effects on learning or memory. We could also test the effect of cocaine, a dopamine transport inhibitor, on dopamine clearance. CNPEs would allow for measurements in discrete areas of the mushroom body as well as other regions of the adult fly brain. The effect of cocaine will vary if dopamine uptake is different in different brain regions.

\subsection{Synaptic Transmission Studies of a Single Synapse}

Currently with CNPEs and CFMEs we are able to study volume transmission of intercellular communication. The synapse between neurons is too small for a CNPE used in this work; therefore, measurements are made in the extracellular space. Studying synaptic transmission and exocytosis is one possible biological application of CNPEs. CNPEs with open tips have been fabricated as small as 10 's of nanometers ${ }^{5}$, and a single synapse is less than $50 \mathrm{~nm}^{10}$. With a cultured neuronal network and future modifications to CNPEs, we could measure dopamine release and uptake in a single synapse. The fabrication method for solid tipped CNPEs would need to be altered to only have a small, polished disk of carbon exposed. I hypothesize this could be done by optimizing the pipette pulling parameters and implementing beveling techniques. Studies have been done with nanoelectrodes and amperometry to monitor dopamine release from single living vesicles. ${ }^{11}$ Using FSCV would be advantageous because we could distinguish neurotransmitters by their cyclic voltammograms. 


\subsection{Final Conclusions}

CNPEs are another viable tool for in vivo measurements of dopamine in

Drosophila. CNPEs have similar electrochemical characteristics comparable to CFMEs; however, there is much room for improvement in size and batch fabrication. CNPEs have exciting applications because of the capability of high spatial resolution measurements. Coupled with FSCV, CNPEs will allow for real-time detection of dopamine in specific brain regions of Drosophila. 


\subsection{References}

1. Takmakov, P.; Zachek, M. K.; Keithley, R. B.; Walsh, P. L.; Donley, C.; McCarty, G. S.; Wightman, R. M. Carbon Microelectrodes with a Renewable Surface. Analytical Chemistry 2010, 82, 2020-2028.

2. Brazell, M. P.; Kasser, R. J.; Renner, K. J.; Feng, J.; Moghaddam, B.; Adams, R. N. Electrocoating carbon fiber microelectrodes with Nafion improves selectivity for electroactive neurotransmitters. Journal of Neuroscience Methods 1987, 22, 167-172.

3. Hashemi, P.; Dankoski, E. C.; Petrovic, J.; Keithley, R. B.; Wightman, R. M. Voltammetric Detection of 5-Hydroxytryptamine Release in the Rat Brain. Analytical Chemistry 2009, 81, 9462-9471.

4. Long, J. T.; Weber, S. G. Noise at Microelectrodes and Microelectrode Arrays in Amperometry and Voltammetry. Analytical Chemistry 1988, 60 (20), 2309-2311.

5. Schrlau, M. G.; Falls, E. M.; Ziober, B. L.; Bau, H. H. Carbon nanopipettes for cell probes and intracellular injection. Nanotechnology 2008, 19 (1).

6. Schrlau, M. G.; Dun, N. J.; Bau, H. H. Cell Electrophysiology with Carbon Nanopipettes. ACS NANO 2009, 3 (3), 563-568.

7. Riemensperger, T.; Voller, T.; Stock, P.; Buchner, E.; Fiala, A. Punishment Prediction by Dopaminergic Neurons in Drosophila . Current Biology 2005, 15, 1953-1960.

8. Pascual, A.; Preat, T. Localization of Long-Term Memory Within the Drosophila Mushroom Body. Science 2001, 294 (5544), 1115-1117.

9. Mao, Z.; Davis, R. L. Eight Different Types of Dopaminergic Neurons Innervate the Drosophila Mushroom Body Neuropil: Anatomical and Physiological Heterogeneity. Front Neural Circuits 2009, 3.

10. Agnati, L.; Guidolin, D.; Guescini, M.; Genedani, S.; Fuxe, K. Understanding wiring and volume transmission. Brain Research Reviews 2010, 64 (1), 137-159.

11. Wu, W.-Z.; Huang, W.-H.; Wang, W.; Wang, Z.-L.; Cheng, J.-K.; Xu, T.; Zhang, R.-Y.; Chen, Y.; Liu, J. Monitoring Dopamine Release from Single Living Vesicles with Nanoelectrodes. J. Am. Chem. Soc. 2005, 127, 8914-8915. 